\title{
Study of contact forces in the bearing part of the protective coating of underground pipelines in gravel and cobbles
}

\author{
R.A. Matsiuk \& V.V. Ulanov \\ Gubkin University, Moscow, The Russian Federation
}

\begin{abstract}
In the construction and operation of underground pipelines, the feasibility of engineering methods of protecting the insulation coating from external mechanical impacts should be based on the existing loads. When an underground pipeline is laid on coarse gravel and very coarse soil, the analysis of the support forces at the base of the pipeline is a pressing issue. In particular, the difficult conditions for the construction, reconstruction, repair and operation of underground pipelines include cases when the pipeline route passes through sections of rocky soils. Discrete rocky conditions include large grain content in the form of coarse gravel, cobbles, and boulders. The article analyzes the influence of the size of foundation soil components on the magnitude of force arising in the protective coating of the support part of an underground pipeline depending on the unevenness of the soil and its granulometric composition. One of the directions for studying the distribution of contact-mechanical interaction in granular soils is a discrete model. The distinction of the discrete medium model is that individual elements of its structure are viewed as mechanically interacting bodies. Structural elements are particles of bulk material that can be considered as spheres. The results of modeling the contact problem using the discrete (granular) model of the base of the pipeline are presented. It was found that with the same diameter size and depth of burial, the force varies depending on the pipe diameter chosen in the project (for a pipe of $1420 \mathrm{~mm}$, the maximum difference was $23 \%$ ). In the case of unevenness of the ground, the force in the supporting part can increase by 3-5 times. The conditions of stability and strength of underground pipelines are formulated taking into account the discreteness of the base particles.
\end{abstract}

\section{Introduction}

Reliability of underground trunk pipelines largely depends on the state of external protection, in particular, on the state of the protective coating of the layer of anticorrosive protection (insulation). In this regard, the study of the basic laws of the interaction of external loads and environmental impacts and conditions with the protective coating of a pipeline is of great importance.

At the stages of loading and transportation, warehousing, welding, installation, testing, and operation, underground pipelines experience a whole range of adverse loads and 
effects. All these operations entail a phased accumulation of coating defects. The prevention of defects ensures the protection of the metal of the pipe from the corrosive environment, its uninterrupted operation, and reduces technological, and financial risks during operation. Coatings for underground trunk pipelines based on liquid thermosetting compositions, extruded polyolefins, heat-shrinkable tapes, epoxy powder coating, coldapplied mastic-polymer tapes, cold-applied adhesive tapes, and hot-applied bitumeninous mastics are currently available. The task of assessing the choice of coating for the specific conditions of the construction site is relevant, especially when the route passes through rocky, crushed stone, and permafrost soils, due to the high hardness of the rocks.

By protective coatings we mean insulating materials and their structures used for underground pipelines to create a barrier that constrains the access of external corrosive media to the metal. The protective effect of coatings is manifested in the reduction of the corrosion rate and, all other things being equal, depends on the insulation coating material, its design, type, including thickness and mechanical characteristics. Over time, the protective effect decreases, therefore long-term and stable functioning of the mechanism of protective action of coatings is a prerequisite for their effectiveness.

Protective coatings must be inert to the metal of the pipeline and the external environment, and also have high dielectric properties significantly slowing down corrosion. To ensure the protection of the pipelines against corrosion, a combination of passive (insulation coatings) and active (electrochemical) protection is used.

Based on grain sizes, international standard (ISO 14688-1:2017) groups soils into sands (Sa, 0.063-2 mm), gravel (Gr, 2-63 mm), cobbles (Co, 63-200 mm), boulders (Bo, 200- 630 $\mathrm{mm}$ ), and large boulders (Lbo, $>630 \mathrm{~mm}$ ). The study considers soil within the gravel $(\mathrm{Gr})$ and cobbles (Co) soil groups. By coarse soil, we mean rock fragments with a predominance of particles larger than $2 \mathrm{~mm}$ (over 50\%). Assessment of the changes to physical soil properties caused by compression of the soil includes soil strength, penetration resistance, degree of aggregation, inter-aggregate porosity, and relative density.

The study considers the applied case where the required amount of finely dispersed filler is not available in the region of construction, and extraction and transportation incur significant economic costs. In this case, there is a need to use local rocky soil as a building material. In the study, the author solves the problem of determining the maximum size of discrete elements of the foundation soil in relation to the underground pipeline.

\section{Object and methods of study}

The object of study is the protective coating of underground pipelines. The study was carried out using theoretical methods based on the analysis of technical requirements for protective coatings, the theory and modeling of the interaction of a discrete (granular) medium of the base of an underground pipeline with its protective coating.

To ensure mechanical protection of the insulation coatings in clastic soil, methods of engineering protection are used, including protective structures and shells that compensate for mechanical loads and impacts during the construction and operation of an insulated underground pipeline, as well as various types of impacts on the granular and filler soil, associated with its replacement with the finely-dispersed ground or its crushing and the use of treated soils (with poisons, hydrophobization, etc.).

According to (SP 36.13330.2012, SP 86.13330.2014), when laying a pipeline in rocky, gravel-pebble, crushed stone, and frozen soils, it is necessary to backfill the foundation composed of soft soils. Soils with a particle size of up to $5 \mathrm{~cm}$ are used for filling as a means of engineering protection of the insulating layer of the underground main pipeline, through backfilling the bottom of the trench with a $10-\mathrm{cm}$ layer of ground surface material. The main disadvantage is the laboriousness of achieving a high degree of compaction of the 
material and achieving a uniform granulometric composition of the bedding soil. In addition to crushing rocky soil, bags or three-dimensional geogrids made of nonwoven material with sand or rock sheets are allowed to be used, and in some cases, continuous concreting of the pipe is used.

Protective structures include three-dimensional geogrids made of interconnected vertically oriented polymer sandbags [1], sandbag foundations s or supports made of various materials (Figure 1), cofferdams preventing soil washout, ballast protection devices, drainage systems, and thermal stabilizers in case of application in permafrost soils, which protect the pipeline coating from the unevenness of the base in case of its thawing. Protective shells include non-woven synthetic materials (rock shield), overall concreting, and using shells and composite rolled materials for the base, wrapping, and rack materials.
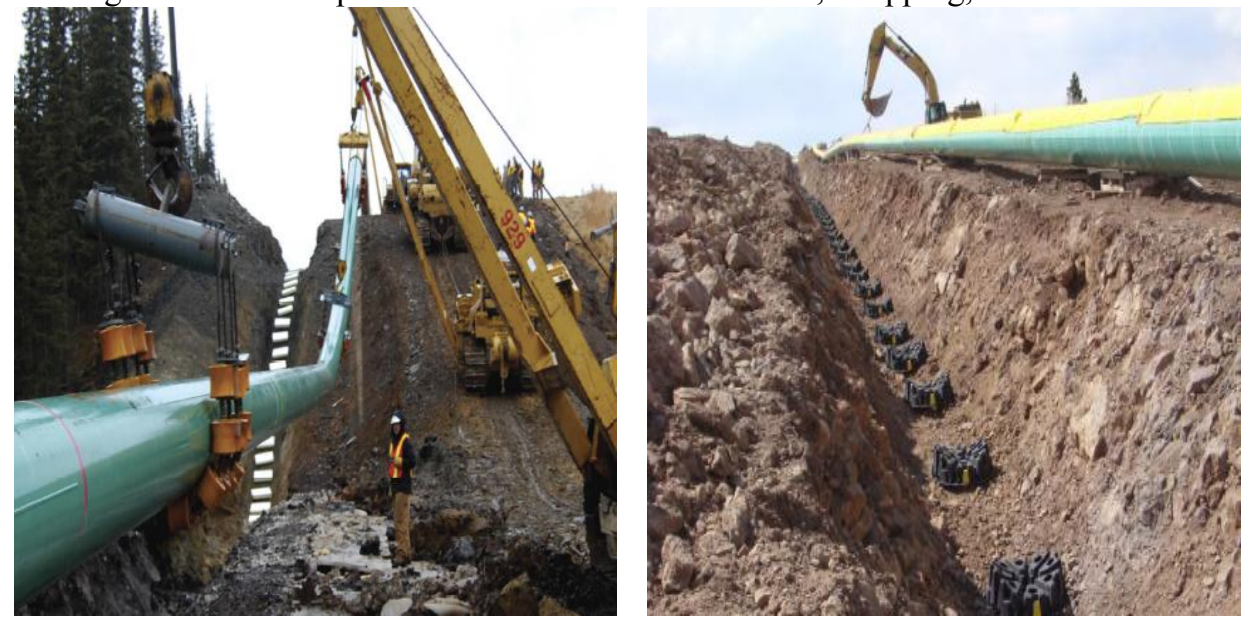

Fig. 1. Polystyrene foam pillows and polymer supports.

As a result of numerous inspections of large-diameter trunk pipelines $(820-1420 \mathrm{~mm})$, it was found that the protective coating of the pipeline can receive perforation damage, both in its supporting and upper parts [1-4]. This specific damage to protective coatings has been demonstrated in the paper [5] on the example of long-term diagnostic studies of the gas pipelines in Western Kazakhstan.

If there are several concentrated or distributed forces that affect the stresses appearing in the bearing part of the pipeline, the law of superposition should be applied. The stress-strain state of the pipeline will be determined by the characteristics of the loads acting it (GOST R 51164-98) [6]. The design of pipeline supports at the bottom of the trench to a large extent determines the magnitude of the compressive stress in the protective coating of the supporting part of the pipeline, arising during its laying and operation.

Consideration of the processes of the interaction of contacting bodies requires the analysis of the conditions of contact loading. In the contact zone, oftentimes, the loading conditions of the material are the most severe, and structural damage processes begin from the surface, and in the case of an underground pipeline, they begin from the surface of the protective coating. Thus, the study of the physicomechanical properties of coatings is an important and critical task.

Assessing the ground load on underground pipes is often a difficult task due to many factors: from the characteristics of soil compressibility, compaction coefficient between the pipe and the sidewall of the trench, to the design decision on the choice of the laying method [7]. The interaction schemes of the protective coating of underground pipelines in gravel and cobbles are presented in Fig. 2. 


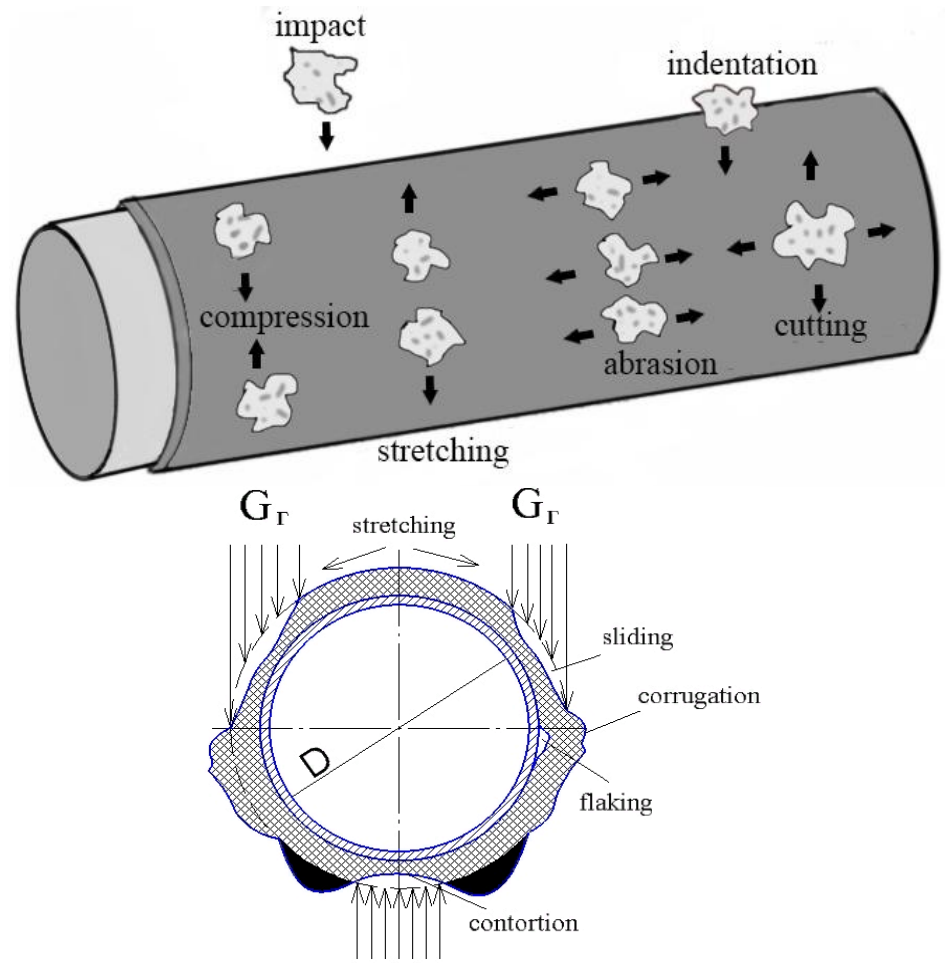

Fig. 2. Impacts and loads on protective coatings of underground pipelines: a) the mechanical effects of particles on the coating; b) the main types of defects in the protective coating during operation

The mechanical impacts that lead to damaging the insulating coatings of underground pipelines in gravel and cobbles can include such damages as indentation, cutting, stretching, compression, abrasion, and other impacts. The influence of these factors in the contact interaction can lead to deterioration of physical and mechanical characteristics of the coating material, its cracking, fracture, as well as a decrease in adhesion to the metal surface of the pipes.

During the construction process, the impact on the coating can occur both during the preparatory stage (storage, loading and unloading, transportation of coated pipes, etc.), and during installation (when lowering the pipe to the walls and the bottom of the trench, during backfilling with clastic material). When a certain level of load is reached or when it is applied repeatedly, destruction can occur in the contact zone of the two contacting bodies. The first local development can lead to a range of damage, from peeling of the coating, crushing of the layer, to through damage and removal of the coating from the pipe. The combination of peeling and through damage is of the most dangerous nature, which contributes to the bilateral destruction of the coating and subfilm corrosion of the pipe metal, with high probability of shielding protective currents of electrochemical protection systems.

When backfilling with coarse-grained soil, the impact energy will depend on the size of the clastic material, especially its mass and the height of the particles falling over the surface of the coated pipeline. For this impact, the norms provide such an indicator as shock resistance. A number of conditions may govern the approach to assessing the degree of impact resistance. The methods of impact resistance tests in the analyzed documents differ in the drop height, the weight of the load, and the rate of passage of the indicator, but at the same time they are carried out according to the same principle: the striker falls from a 
height onto the coating surface. Control of the indicator is carried out by checking the coating for the absence of destruction of the coating after the impact.

Another mechanical effect is the stretching of the coating in the upper generatrix, which can cause the rupture of the material. Such an effect occurs during the compaction of the backfill soil between the pipe and the walls of the trench in the course of operation. For this impact, the norms provide such an indicator as "elongation at break". The test method for elongation at break refers directly to the material of the upper protective layer, but not to the structure as a whole, which is not entirely correct. The test is carried out on the material itself without taking into account the geometry of the pipe (denting, bending, etc.) and the construction of the coating.

At the All-Union Research Institute for the Construction, Operation of Pipelines and Fuel and Energy Complex Objects [8], the dependence of the angle of support of the pipeline (knowing which it is easy to calculate the area of the support) on the diameter of the pipeline and the vertical load on various pastels was studied. As a result of those studies, it was found that the angle of support does not depend on the diameter of the pipes. The research results are presented in Figure 3 as a function of the angle of support from the linear load.
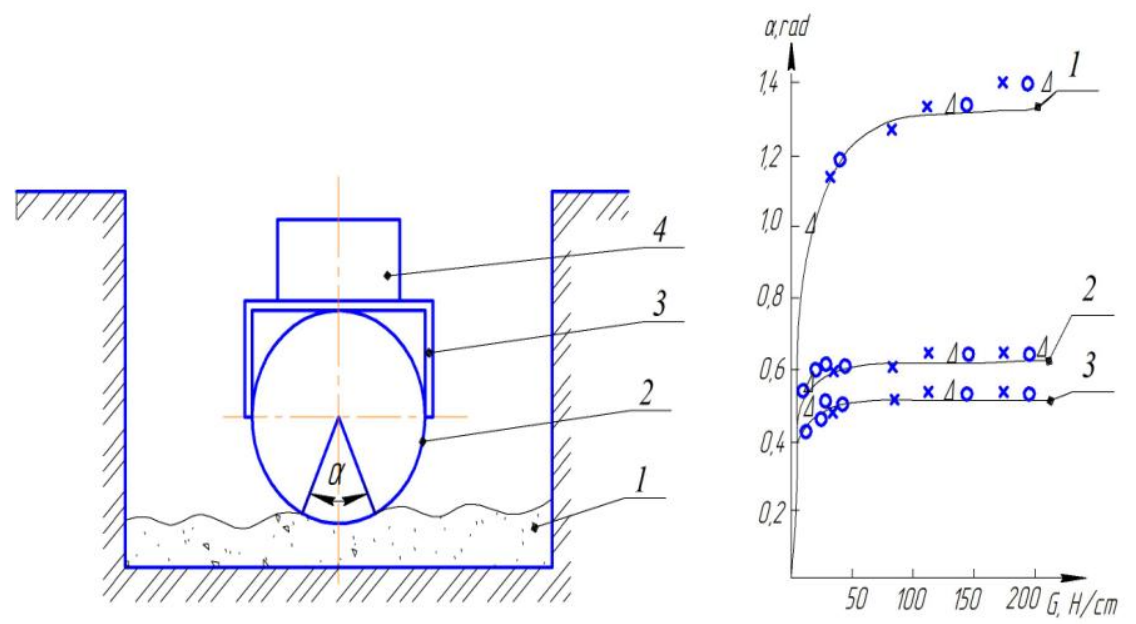

Fig. 3. The dependence of the angle of support of the pipeline in various soils from the linear load: 1 clay bottom of the trench, flooded with water; 2 - loosened clay, loess; 3 - sand; diameter of pipes, $\mathrm{mm}: \mathrm{x}-1420 ; \mathrm{O}-1220 ; \Delta-820 ;-377$.

It can be seen from the figure that this dependence is asymptotic, and the results obtained on pipes of different diameters can be described by a single curve; the results of the experiments conducted in loess and clay soils are very close, which suggests the validity of the established dependence for other soils (sandy loam, loamy soil, chernozem).

A characteristic feature of the performance of the shell in an elastic medium is the presence of a squeezing layer of the medium involved in a joint performance with the shell $[8,9,10]$. Under the impact of the vertical compressive force onto the insulation coating, normal compressive stress and tangential shear stresses arise. Consequently, the calculation is based on taking into account the joint contact-mechanical interaction of the shell of the protective coating of the underground pipeline and the environment $[11,12,13,14]$.

One of the directions of studying the distribution of contact-mechanical interaction in granular soils is the model of discrete media. The peculiarity of the discrete medium model is the consideration of the individual elements of its structure as mechanically interacting bodies. The structural elements are particles of bulk material that can be represented as spheres. The most broadly describing work, covering both stresses and strains in granular 
media, are the works of I.I. Kandaurov [15, 16, 17] suggesting a description of the operation of granular materials based on probabilistic methods. The theory of mechanics of granular media was developed by E. Litvinishen [18], J. Litwiniszyn [19, 20, 21, 22, 23] and others, but they did not achieve any serious progress in the theory of granular media.

Let's consider the load distribution between the bedding soil and the underground pipeline coating shown in Figure 4. In this case, the parameters of the compressive load in the lower part of the pipeline are considered to be the sole weight of the pipeline, the force of the vertical pressure of the soil, the weight of the transported product, the weight of the insulation and coatings, as well as the impact of transport from the soil surface on the pipeline. Earlier, in the papers $[24,25,26]$, the issue of determining the resultant uniaxial compression force $\mathrm{G}$ was considered. The sole weight of the pipeline, the force of the vertical pressure of the soil, the weight of the transported product, and the weight of the insulation were considered as the parameters affecting the compressive load.

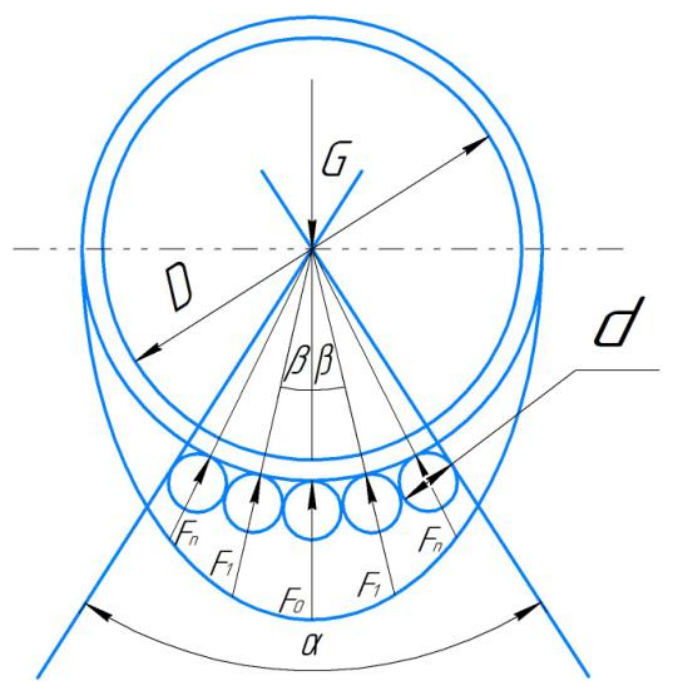

Fig. 4 Scheme of the distribution of the load between the bedding soil and the underground pipeline coating.

It is assumed that the pipeline is covered with soil and is located at a depth of H. Let us set the condition for the equilibrium of the system:

$$
\begin{aligned}
& G=F_{0}+2 F_{1} \cos \beta+2 F_{2} \cos 2 \beta+2 F_{3} \cos 3 \beta+\ldots . .+2 \mathrm{~F}_{\mathrm{n}} \cos \mathrm{n} \beta, \\
& F_{1}=F_{0} \cos ^{\frac{3}{2}} \beta ; F_{2}=F_{0} \cos ^{\frac{3}{2}} 2 \beta ; F_{3}=F_{0} \cos ^{\frac{3}{2}} 3 \beta \ldots F_{n}=\mathrm{F}_{0} \cos ^{\frac{3}{2}} \mathrm{n} \beta .
\end{aligned}
$$

Then

$$
\begin{gathered}
F_{0}=\frac{G}{\left(1+2 \cos ^{\frac{5}{2}} \beta+2 \cos ^{\frac{5}{2}} 2 \beta+2 \cos ^{\frac{5}{2}} 3 \beta \ldots .2 \cos ^{\frac{5}{2}} n \beta\right)}, \\
\beta=2 \arcsin \frac{d}{2\left(\frac{D}{2}+\frac{d}{2}\right)},
\end{gathered}
$$


where $\mathrm{G}=$ vertical compressive force, $\mathrm{N} ; \mathrm{D}=$ diameter of the pipeline, $\mathrm{m} ; \mathrm{d}=$ diameter of the soil particles, $m ; \beta=$ angle between the point of contact of the particle and the vertical axis of the pipeline, ${ }^{\circ} ; F_{0}, F_{1}, F_{2}, F_{3} \ldots F_{n}=$ reaction forces in the contact of soil particles and coatings, $\mathrm{H}$.

Equation (1) includes only those members for which the angle $n \beta$ will be in the assumed support area; the angle itself is the complete angle $\alpha=\mathrm{n} \beta$ (approximately $27^{\circ}-32^{\circ}$ for largediameter pipes) since we are interested exactly in the reaction of the supporting part of the bed of the pipeline.

\section{Results}

Figure 5 shows the results of the analysis of the possible types of the unevenness of support in the base of the underground pipeline in the trench in both longitudinal and transverse directions. The most dangerous combination was identified.

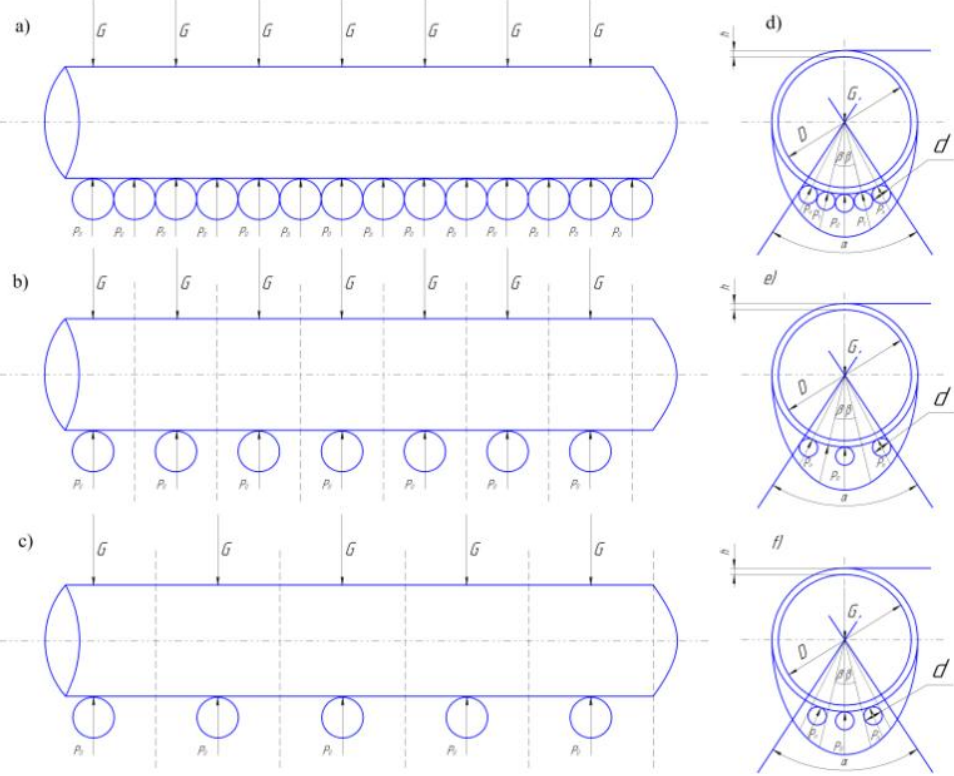

Fig. 5. Patterns of uneven foundations for underground pipelines in the trench: a) and d) uniform foundation in the longitudinal and transverse directions, respectively; b) and c) are the variants of unevenness in the longitudinal direction; e) and f) are the variants of unevenness in the transverse direction.

The main reasons for the formation of uneven bases from crushed rocks, crashed stone or permafrost soil can include: the difficulty of achieving uniformity of the granulometric composition of the soil (same particle size); spalling of less durable particles during laying and backfilling; the difference in the surface configuration of particles and their tendency to take the most stable position; the tendency of the structure to lean on the hardest and protruding particles; soil removal from the base by surface waters when there is a significant slope, etc.

Considering in more detail the schemes of possible irregularities at the base of the bottom of the trench of an underground pipeline, we can conclude that a combination of schemes c) and e) will be the most hazardous.

Based on the presented solution, it is possible to formulate the following condition for the stability of the pipeline foundation with the uniformity of the granulometric 
composition of the crushed coarse soil: the maximum size of soil particles in the foundation soil at a given angle of support must ensure at least three points of contact with the coating. And this means that for small-diameter pipes (114-121 mm), the maximum particle size of the base filling should be $10-12 \mathrm{~mm}$ in diameter.

The direction of the normal of the support force to the particles should not exceed the angle of internal friction $\varphi$ of the clastic material. The angle of internal friction $\varphi$ of the clastic material characterizes the maximum angle of application of the force at which the stability of the particles in the backfill is disturbed, i.e., the resistance of soil particles to the shift:

$$
n \beta<\varphi
$$

It was proposed to distinguish between rigid and non-rigid protective coatings and shell. Rigid coatings affect the ring stiffness of the pipeline structure. It is possible to formulate the following conditions for the stability of the base of the pipeline with the uniformity of the granulometric composition of the crushed bedding:

1. For non-rigid coatings - the maximum size of soil particles in the backfill at a given angle of support must ensure at least three points of contact with the coating.

2. For rigid coatings - a two-point support system is allowed at a given angle of support and compliance with strength conditions.

3 . The direction of the normal of the bearing force to the particles should not exceed the angle of internal friction $\varphi$ of the clastic material: $n \beta<\varphi$.

The difference in the adopted support schemes is explained by the different in terms of the bearing capacity of each of the materials and structures of the pipe protection.

The angle of internal friction $\varphi$ of the clastic material characterizes the maximum angle of application of the force at which the stability of the particles in the backfill is disturbed, i.e. resistance of soil particles to shear.

We can formulate the conditions of stability:

1. The calculated compressive force of particles at the base should not exceed the force of destruction of particles under uniaxial compression:

$$
G x<P p \text {, }
$$

where $\mathrm{Gx}$ is the calculated compressive force of particles in the base, $\mathrm{N}$;

$\mathrm{Pp}$ is the force of destruction of the particles in uniaxial compression, $\mathrm{N}$.

2. The calculated compressive force of particles in the base should not exceed the ultimate strength of the protective coating or shell (in the presence of mechanical protection) in accordance with the tests carried out on the protective material:

$$
G x<P m \text {, }
$$

where Pm is the force of destruction of the material of the protective coating, N.

3. The calculated vertical load on the upper generatrix must ensure the lateral stability of the pipeline structure with a coating and/or a shell, i.e. resist the formation of an unacceptable level of ovalization.

4. The contact force should not lead to local deformation, i.e. violation of the shape of the structure - the formation of dents in the pipes. In the case of a containment shell, it is necessary to assess the effect of its rigidity on the stability of the structure.

Thus, provided that the calculated compressive force of the particles in the base of the underground pipeline exceeds the force of destruction of the particles in uniaxial compression, the size of the largest fraction of the foundation soil should be reduced. The same conclusion applies to the case when the ultimate strength of the coating exceeds the calculated one. A more durable protective covering should be chosen or the size of the foundation soil particles should be reduced. Figure 6 shows the results of calculating the force in the protective coating of an underground pipeline at a depth of $1.2 \mathrm{~m}$ with a pipe diameter of $1420 \mathrm{~mm}$. 


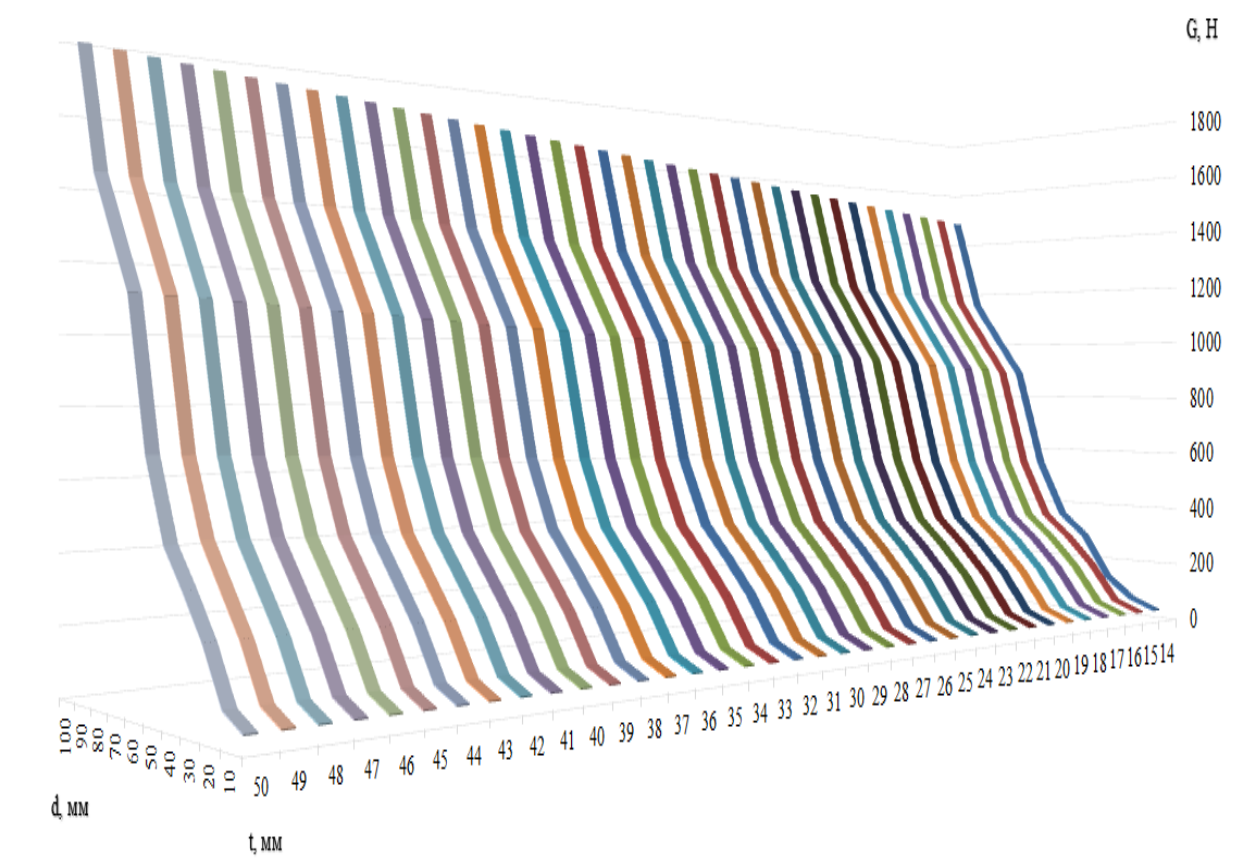

Fig. 6. The dependence of the generated force in the protective coating of the underground pipeline on the size of the soil particles of the filling $d$ and the pipe wall thickness $t$ (the pipe diameter equals $1420 \mathrm{~mm}, \mathrm{H}=1.2 \mathrm{~m}$ ).

The force values are indicated depending on the size of the main particles in the filling and the thickness of the pipeline wall. Under equal conditions, namely, the depth of the pipeline, the size of the particles of the filling, and the material of the pipe, the difference between the forces created at minimum wall thickness and maximum wall thickness was about $22 \%$.

When laying underground pipelines in the ground composed of particles of rocky, gravel, cobbles, and frost soils, their hardness will be a significant indicator of the quality of the pipeline insulation. This issue requires a more detailed consideration in terms of changing operational efforts. The solution to this issue can significantly expand the range of materials used to protect underground pipelines. The author is developing a methodology for assessing the required hardness for protective coatings and shells for underground cylindrical structures.

\section{Conclusions.}

When evaluating the protective coating or structure, it is necessary to take into account the change in the value of the compressive forces in the base of the pipeline, depending on its diameter, wall thickness, depth of burial, and the granulometric composition of the base soil.

If there are irregularities in the base of the underground pipeline according to schemes c) and e) (Fig. 5) when laying in rocky, crushed stone, and permafrost soils, the force in the coating can increase by up to 5 times. Depending on the choice of the wall thickness for pipes of large diameter and ceteris paribus, the difference in the created force in the foundation can reach $22 \%$. The conditions of stability and strength of underground pipelines are formulated taking into account the discreteness of the base particles. When using coarse gravel and cobbles in the base of the pipeline, it is necessary to check whether 
this will create dents in the pipe and also check for the stability of the annular section to external loads.

Thus, the developed methodology for assessing the contact forces in the supporting part of underground pipelines in gravel and cobbles can be applied both to protective coatings and to shell protective structures (concrete coating, rock sheet, lining with a polymer rail, etc.). The choice of the engineering protection method for coatings and shells should take into account the perceived loads from the foundation.

\section{References}

1. G.G. Vasiliev, M.A. Lezhnev, E.N. Gainulin, Laying pipelines on permafrost soils using soil modules, Science and technology of pipeline transport of oil and gas. Moscow: Pipeline Transport Institute, LLC 3: 12-17 (2011)

2. V.I. Voronin, T.S. Voronina, Insulating coatings of underground oil pipelines (Moscow: VNIIOENG All-Russian Research Institute of the Organization for the Management of the Economy of the Oil and Gas Industry, 1990).

3. R.R. Gizzatullin, Improving the Methods of Protecting Main Pipelines from Corrosion in Field Conditions based upon Newly Developed Insulating Materials (Synopsis of the Doctoral Thesis in Engineering Sciences, Ufa, 2004)

4. V.A. Popov, E.S. Lukin, A.I. Istomin, Methods and technical means of quality control of protective coatings of oil and gas industry objects at different stages of their life cycle, Corrosion of the territory of oil and gas, 6: 42, (2013).

5. R.R. Khasanov, Problems of protective coatings for pipelines on the example of the trunk pipelines of Western Kazakhstan, Transport, Storage of Oil and Gas, 12 (1): 97 102, (2014).

6. S.V. Vinogradov, Calculation of external loads for underground pipelines (Moscow: Stroyizdat, 1980)

7. T. Löffler, New Field-Applied Anti-Corrosion System for Pipelines with Moist Surfaces, Novel Construction Fall Plenary Session Geneva, 2016

8. V.K. Semenchenko, Insulation on pipelines: Collection of scientific works, (Research Institute for the Construction of Trunk Pipelines. Moscow. VNIIST, 1982)

9. E.E. Brykov, The use of geosynthetics to protect the external anti-corrosion coating of pipelines from mechanical damage, Pipeline transport: theory and practice, 1(11): 38 45 (2008)

10. I.I. Veliulin, R.R. Khasanov, The effect of different types of insulation coatings on the resistance of gas pipelines to stress-corrosion processes, Territory NEFTEGAS, 1-2: 52-56 (2019)

11. R.A. Matsiuk, Evaluation of the effectiveness of testing of protective coatings of pipelines through penetration inspection, Abstracts of the IX International Scientific and Technical Conference "Reliability and safety of the main pipeline transport": 51-52, (2018)

12. M.L. Medvedeva, A.V. Muradov, A.K. Prygaev, Corrosion and protection of trunk pipelines and reservoirs: a training manual for universities of oil and gas profile. (Moscow: Gubkin Russian State University of Oil and Gas, 2013)

13. H. Pouraria, J.K. Sea, J.K. Palk, CFD Simulation of the effect of different oils on water wetting and internal corrosion of oil pipelines, Proceedings of the ASME 35th international conference on ocean, offshore and arctic engineering, 5, UNSP V005T04A023 (2016) 
14. D. Shantanu, S. Shibau, A review on different pipeline fault detection methods, $J$ of Loss Prevention in the Process Industries, 41: 97-106. (2016).

15. I.I. Kandaurov, Theory of Discrete Distribution of Stress and Deformation (Izd. VATT, 1959).

16. I.I. Kandaurov, Mechanics of granular media and its application in construction. (Leningrad: Stroyizdat, 1966).

17. I.I.Kandaurov, Mechanics of granular media and its application in construction (Leningrad: Stroyizdat, 1988).

18. E. Litvinishen, Displacement of bulk media as a stochastic process, Bulletin of the Polish Academy of Sciences, 4 (1955)

19. J. Litwiniszyn, Stochastic methods in mechanics of granular bodies (Wien: Springer, 1974).

20. R.A. Muller, Deformation of granular soil, Proc. All-Union Sci.-Res. Mining Inst. Leningrad (1963).

21. R.A. Muller, To the statistical theory of stress distribution in a granular soil foundation, Bases, foundations, and soil mechanics, 4 (1962)

22. W. Brzakala, On the propagation of shear stress in Kandaurov granular medium, Bulletin of the Polis Academy of sciences, Technical sciences, 36 (7-9): 407-413. (1988).

23. S.J. Matysiak, P. Pusz, Axisymmetric Boussinesq problem for granular half-space Bulletin of the Polish Academy of sciences, Technical Sciences, 33 (7-8): 351-358 (1985).

24. R.A. Matsiuk, Evaluation of the influence of the uneven base on a protective coating of underground pipeline, Pipeline transport: theory and practice, 6 (64): 56-59 (2017).

25. R.A. Matsiuk, Research of conditions of supporting underground pipeline and loads on protective coating, Oil and Gas Studies, 4: 130-139 (2019)

26. R.A. Matsiuk Analysis of the Requirements of Foreign and Domestic Regulatory and Technical Documents for Protective Coatings of Underground Pipelines in Coarse Clastic Soils, Oil and Gas Territory, 12: pp. 46-54 (2019). 